\title{
Patient outcomes in idiopathic scoliosis are associated with biological endophenotypes: 2020 SOSORT award winner
}

\author{
Marie Beauséjour ${ }^{1,2,3} \cdot$ François Vaillancourt $^{1,4} \cdot$ Marie-Yvonne Akoume $^{7}$. Anita Franco ${ }^{8} \cdot$ Stefan Parent ${ }^{1,2,3}$. \\ Hubert Labelle ${ }^{1,2,5}$. Julie Joncas ${ }^{1,3} \cdot$ Frédérique Desbiens-Blais $^{1,6}$. Jean-Marc Mac-Thiong ${ }^{1,2,3}$. \\ Marjolaine Roy-Beaudry ${ }^{1}$. Carl-Éric Aubin ${ }^{1,6} \cdot$ Alain Moreau $^{8,9,10}$
}

Received: 17 August 2020 / Revised: 17 August 2020 / Accepted: 20 August 2020 / Published online: 29 August 2020

(c) The Author(s) 2020

\begin{abstract}
Purpose Bracing is the treatment of choice for idiopathic scoliosis (IS), unfortunately factors underlying brace response remain unknown. Clinicians are currently unable to identify patients who may benefit from bracing, and therefore, better molecular stratification is critically needed. The aim of this study is to evaluate IS patient outcomes at skeletal maturity in relation to biological endophenotypes, and determine specific endophenotypes associated to differential bracing outcomes. This is a retrospective cohort with secondary cross-sectional comparative studies.

Methods Clinical and radiological data were collected from 563 IS patients, stratified into biological endophenotypes (FG1, FG2, FG3) based on a cell-based test. Measured outcomes were maximum Cobb angle at skeletal maturity, and if severe, spinal deformity $\left(\geq 45^{\circ}\right)$ or surgery was attained. Treatment success/failure was determined by standard progression thresholds $\left(\mathrm{Cobb} \geq 45^{\circ}\right.$ or surgery; Cobb angle progression $\geq 6^{\circ}$ ). Multivariable analyses were performed to evaluate associations between endophenotypes and clinical outcome.

Results Higher Cobb angles at maturity for FG1 and FG2 patients were observed ( $p=0.056$ and $p=0.05)$, with increased likelihood of $\geq 45^{\circ}$ and/or surgery for FG1 (OR $=2.181$ [1.002-4.749] and FG2 (OR=2.141 [1.038-4.413]) compared to FG3. FG3 was 9.31 [2.58-33.61] and 5.63 [2.11-15.05] times more likely for bracing success at treatment termination and based on the $<6^{\circ}$ progression criterion, respectively, compared to FG1.

Conclusion Associations between biological endophenotypes and outcomes suggest differences in progression and/or bracing response among IS patients. Outcomes were most favorable in FG3 patients. The results pave the way for establishing personalized treatments, distinguishing who may benefit or not from treatment.
\end{abstract}

Keywords Idiopathic scoliosis $\cdot$ Bracing $\cdot$ Spinal deformity progression $\cdot$ Biological endophenotypes $\cdot$ Treatment outcome

Alain Moreau

alain.moreau@recherche-ste-justine.qc.ca

1 Sainte-Justine University Hospital Research Center, Montreal, QC, Canada

2 Department of Surgery, Faculty of Medicine, Universite de Montréal, Montreal, QC, Canada

3 Department of Community Health Sciences, Faculty of Medicine and Health Sciences, Université de Sherbrooke, Longueuil, QC, Canada

4 Department of Surgery, Faculty of Medicine and Health Sciences, Université de Sherbrooke, Sherbrooke, QC, Canada

5 Orthopedic Division, Sainte-Justine University Hospital, Montreal, QC, Canada
6 Polytechnique Montréal, Montreal, QC, Canada

7 Department of Pharmacology and Toxicology, Faculty of Medicine, Université des Sciences de la Santé (USS) de Libreville, Libreville, Gabon

8 Viscogliosi Laboratory in Molecular Genetics of Musculoskeletal Diseases, Sainte-Justine University Hospital Research Center, Université de Montréal, Montreal, QC, Canada

9 Department of Stomatology, Faculty of Dentistry, Université de Montréal, Montreal, QC, Canada

10 Department of Biochemistry and Molecular Medicine, Faculty of Medicine, Université de Montréal, Montreal, QC, Canada 


\section{Introduction}

Spinal deformities, scoliosis in particular, represent the most prevalent type of orthopedic deformity in the pediatric population while idiopathic scoliosis represents the most common form of scoliosis [1]. Juvenile idiopathic scoliosis is classically defined as scoliosis first diagnosed between the ages of 4 and 10. This category comprises about $10-15 \%$ of all idiopathic scoliosis in children. Adolescent idiopathic scoliosis (AIS), defined by a clinical diagnosis made between the ages of 10 and 18 years [1], affects an average of $4 \%$ of the global pediatric population. One out of every six children diagnosed with AIS will have a progressive curve requiring active treatment, and there is a predominance of females among the severe cases. The current recommended management of the disease consists of observation (for mild deformities: between $10^{\circ}$ and $24^{\circ} \mathrm{Cobb}$ angle); bracing (for moderate curves: $25^{\circ}-44^{\circ}$ Cobb angle); and spinal surgery (for severe, progressive curves: $\geq 45^{\circ}$ Cobb angle) as a last resort.

Bracing is the primary method for treating moderate scoliosis during the developmental phase of growth. The most promising evidence showed that $72 \%$ of braced patients avoided surgical recommendations in comparison with $48 \%$ of the AIS patients in the observational group [2]. In addition, the BrAIST study reported a positive correlation between the increased duration of brace wear and the success rate of bracing [2]. Up to $30 \%$ of AIS patients cannot be stabilized by brace treatment since factors underlying brace response remain poorly understood.

Despite intensive research efforts, there is no proven method or test available to identify symptomatic individuals, at the earliest stage possible, who are at risk of progression or who may require brace treatment to prevent scoliosis progression [1]. Although a genetic component is recognized, a clear understanding of AIS etiology is still lacking [1-5]. Given the apparent genetic and clinical heterogeneity of AIS, and the limited success of genomics to find definitive causal genes, other approaches need to be applied [6]. In complex multifactorial disorders, such as AIS, biological endophenotypes have the potential utility both in identifying risk genes and in enlightening the pathophysiology. We previously identified three distinct biological endophenotypes for AIS based on a differential protein $\mathrm{G}$ inhibitory (Gi) signaling dysfunction in AIS patients [7-11]. These observations led to the classification of patients into three biological endophenotypes or "functional groups" (referred to as FG1, FG2, and FG3), based on the maximal Gi response observed after Gi alpha subunits stimulation [11]. This molecular classification is very specific given the differential hypofunctionality of the three $\mathrm{Gi}$ alpha subunits occurring in each AIS endophenotype while such patterns are not observed among healthy controls [11]. With respect to AIS development and its nonsurgical treatments (e.g., bracing), the importance of biomechanics $[12,13]$ and bodily responses to mechanical stimuli [14] is generally well established and holds many possibilities for novel personalized therapeutic options. Nevertheless, the mechanisms by which biomechanical cues may affect cellular functions or bracing response, as well as how the sensing apparatus and transduction of mechanical signals operate in AIS pathogenesis, remain poorly understood. Among the known components of the mechanotransduction pathway, G protein-coupled receptors (GPCR) [15] and more specifically Gi-coupled receptor signaling attracted our attention given our previous findings [11]. The objective of this study was to determine if distinctive biological endophenotypes among AIS patients could be associated with a differential outcome at maturity.

\section{Material and methods}

\section{Study population}

This is a one-center retrospective cohort study with doubleblinded data collection concerning IS patients. The inclusion criteria concerned children aged from 5 to 18 years old at their initial appointment at the scoliosis clinic, confirmed or suspected IS (Juvenile JIS or Adolescent AIS) diagnosis, and an availability of a blood sample on which a spectroscopy has been performed to determine the endophenotype. Eligible patients should also have a complete full spine $\mathrm{X}$-ray examination at skeletal maturity (Risser sign of 4 or 5), as suggested by the Scoliosis Research Society [17]. The patients were recruited from June 1996 to August 2011, and data collection was completed in June 2016.

To further assess bracing outcomes, a subsample of patients was identified from the cohort if they reached brace treatment termination during the specified observation period. The patient selection criteria for the secondary cross-sectional comparative study on brace outcomes were: a history and physical examination consistent with AIS diagnosis, and at the time of treatment initiation, a curve between $20^{\circ}$ and $40^{\circ}$ Cobb angle in the coronal plane, a Risser sign between 0 and 2 , no prior treatment, and, either premenarchal or less than one year post-menarchal (in female patients) $[2,16,17]$. For patients with multiple curves, the magnitude of the maximum Cobb angle was used for selection and analysis. 


\section{Biological endophenotypes determination}

\section{PBMC Preparation}

Blood samples obtained from the IS patients were collected in EDTA-treated blood collection tubes. Peripheral blood mononuclear cells (PBMCs) were isolated from whole blood using Ficoll-Plaque (GE Healthcare, ON, Canada), based on the manufacturer's instructions. Isolated PBMCs were preserved in liquid nitrogen until thawed and assayed.

\section{Cellular dielectric spectroscopy (CDS) assay}

All participants were classified according to their biological endophenotype, FG1, FG2, or FG3. The endophenotypes were measured by CDS using PBMCs as previously described $[9,10]$.

\section{Patient outcomes at maturity}

The primary outcome is the maximal Cobb angle at skeletal maturity (Risser 4 or 5 if no X-rays taken at Risser 4) as main Cobb angle measurement is a reproducible clinical index used to describe scoliosis severity. Moreover, this outcome showed some very interesting results in the previous work from which this study is inspired $[4,5]$. The secondary outcome describes the state of the patient who has reached skeletal maturity, and has or has not progressed to a severe Cobb angle $\left(45^{\circ}\right.$, a usual threshold for considering surgery) or undergone corrective surgery (surgical treatments consisting of posterior fusion and instrumentation, or tethering). This outcome, the severity threshold outcome, is used in standardized bracing studies to express failure of the non-surgical treatment [5]. In fact, patients with curves of $45^{\circ}$ or more have a much higher risk of developing functional disabilities caused by their scoliosis, as post-maturity progression is known to be faster when the curvature at maturity is severe or superior to $30^{\circ}$ [6-9].

\section{Brace outcomes at treatment termination}

Brace treatment success is defined as attaining skeletal maturity (Risser sign 4 or 5) with a final Cobb angle $\leq 45^{\circ}$ or not requiring surgery. We also tested curve progression as measured by the difference between the Cobb angle at maturity and the Cobb angle at treatment initiation; success is defined as a Cobb angle progression $<6^{\circ}[16,17]$.

\section{Data collection and statistical analysis}

The patient records composed of multiple electronic platforms were reviewed for eligible participants. Extraction and computation of all radiographic and clinical variables were made by three independent evaluators (a research nurse, a medical student, and an orthopedic surgeon) who were unaware of the endophenotype classification. In addition, endophenotype characterization was performed by research laboratory staff who were unaware of patient outcomes. The documented variables were: age, sex, body mass index (BMI), curve type, Risser score, follow-up duration, and Cobb angles. Additional studied variables describing brace treatment were the treatment duration, the appropriateness of the prescription according to the SRS criteria for AIS [2] and the main type of brace used. The initial state variable distribution among endophenotype groups was verified using $\chi^{2}$ and Kruskal-Wallis/ANOVA tests. Bivariate associations between clinical variables and patients outcomes at maturity were investigated in order to identify potential control variables in the multivariable analyses. Multivariable modeling using linear regression for the Cobb angle at maturity continuous outcome, and using logistic regression for the reach of $45^{\circ}$ or surgery binary outcome, were conducted, both testing for relevant control variables.

For the secondary analysis on brace treatment outcomes, cross-sectional analyses of variable associations were investigated by Log linear models on categorical data as well as $\chi^{2}$ tests. Logistic regression models were performed for the odds ratio calculation in order to describe associations between the bracing outcomes (independently for the $45^{\circ}$ threshold and the $6^{\circ}$ progression criteria) and the endophenotype group, adjusting for other initial state variables at the time of brace prescription. All analyses were performed in IBM SPSS Statistics 20 (64 bits), using a statistical significance level of $\alpha=0.05$.

\section{Results}

\section{Study population}

Over the observation period, 767 young patients were seen for a first visit in scoliosis clinic for IS suspicion. Of this number, 204 were discharged or received no follow-up. Five hundred and sixty-three patients completed their follow-up to maturity in our clinic over the observation period. The clinical and demographic characteristics of the IS patients are described in Table 1. Respectively, 121, 182, and 260 patients were classified as FG1, FG2, and FG3 endophenotype. As expected, the study sample was composed of a higher percentage of females $(83.8 \%, n=472)$ when 
Table 1 Clinical and demographic data of AIS participants at the first visit $(n=563)$

\begin{tabular}{llllll}
\hline Patient characteristics & All participants & FG1 & FG2 & FG3 & $p$ value \\
\hline$N$ & 563 & 121 & 182 & 260 & \\
Age & $13.3 \pm 2.1$ & $13.3 \pm 2.1$ & $13.6 \pm 2.2$ & $13.2 \pm 2.1$ & 0.258 \\
BMI & $19.0 \pm 2.8$ & $18.5 \pm 2.6$ & $19.1 \pm 2.7$ & $19.1 \pm 19.1$ & 0.184 \\
Cobb angle $\left(^{\circ}\right)$ & $15.2 \pm 9.8$ & $21.5 \pm 10.6$ & $22.3 \pm 9.4$ & $20.3 \pm 9.6$ & 0.108 \\
Sex & & & & 0.075 \\
Female & $472(83.8 \%)$ & $102(84.3 \%)$ & $161(88.5 \%)$ & $209(80.4 \%)$ & \\
Male & $91(16.2 \%)$ & $19(15.7 \%)$ & $21(11.5 \%)$ & $51(19.6 \%)$ & \\
Curve type & & & & 0.933 \\
Thoracic & $231(41.3 \%)$ & $47(39.2 \%)$ & $73(40.3 \%)$ & $111(43.0 \%)$ & \\
Thoracolumbar & $197(35.2 \%)$ & $45(37.5 \%)$ & $63(34.8 \%)$ & $89(34.5 \%)$ & \\
Lumbar & $131(23.4 \%)$ & $28(23.3 \%)$ & $45(24.9 \%)$ & $58(22.5 \%)$ & \\
Risser score & & & & \\
0 & $224(39.8 \%)$ & $53(43.8 \%)$ & $60(33.0 \%)$ & $111(42.7 \%)$ & \\
1 & $54(9.6 \%)$ & $10(8.3 \%)$ & $17(9.3 \%)$ & $27(10.4 \%)$ & \\
2 & $48(8.5 \%)$ & $10(8.3 \%)$ & $19(10.4 \%)$ & $19(7.3 \%)$ & \\
3 & $66(11.7 \%)$ & $21(17.4 \%)$ & $21(11.5 \%)$ & $24(9.2 \%)$ & \\
Mature (4 or 5) & $171(30.4 \%)$ & $27(22.3 \%)$ & $65(35.7 \%)$ & $79(30.4 \%)$ & \\
Length of the follow-up (months) & $30.4 \pm 20.7$ & $30.3 \pm 20.4$ & $28.3 \pm 20.7$ & $31.9 \pm 20.7$ & 0.304 \\
\hline
\end{tabular}

compared to males $(16.2 \%, n=91)$. The average age of the patients at first visit was 13.3-year old, and the average initial Cobb angle of the main curve was $21.2^{\circ}$.

\section{Bivariate analysis on data at first visit}

For each variable on initial appointment, the biological endophenotypes were compared to insure uniformity within the variable (Table 1). No differences were found between the biological endophenotypes within the clinical variables of initial Cobb angle of main curve $(p=0.179)$, Risser score $(p=0.085)$, curve type $(p=0.933)$, and brace treatment prescribed ( $p=0.131)$. They were found to be initially uniformly distributed among endophenotypes, just like age, sex, and BMI (Table 1). Furthermore, the length of the follow-up, as defined as the time elapsed between the initial visit and the last visit, was the same for all patients independently of their endophenotype.

\section{Clinical outcomes}

Bivariate analysis provided evidence of more severe health outcomes at maturity in IS patients classified in FG1 endophenotype compared to patients classified in FG3 endophenotype. In fact, the omnibus F test on the three endophenotype groups displayed a difference in Cobb angle of the main curve at maturity between the groups. FG3 group was the less likely to reach high Cobb angles, with an average value of $23.9^{\circ}$ compared to $26.3^{\circ}$ for $\mathrm{FG} 2$ and $27.0^{\circ}$ for FG1 $(p=0.030)$. In addition, the percentage of IS patients who reached a Cobb of $\geq 45^{\circ}$ and/or had a spinal fusion was almost two times higher in the FG1 group with $21.2 \%$, when compared to the FG3 group with $11.2 \%$ and to the FG2 group with $18.6 \%(p=0.024)$.

In multivariable analyses (Table 2), the linear relationship between the Cobb angle at maturity and the biological endophenotypes was borderline significant $(p=0.056$ and $p=0.05$ ) after adjusting for the following control variables in the final model (that appeared to be independently associated with the outcome): Risser sign, initial main curve Cobb angle, type of treatment, and age at maturity. The final model led to an adjusted $R^{2}=0.711$ The initial age, initial BMI, BMI at maturity, length of the follow-up, appropriateness of the brace prescription, and length of the treatment were not associated with the outcome.

Table 3 displays the results of the logistic regression for the reach of final Cobb angle to $45^{\circ}$ or the occurrence of surgery. The multivariable analyses indicated that IS patients classified into FG1 or FG2 endophenotype were 2 times more likely (FG1: $\mathrm{OR}=2.181, p=0.049)$ and $\mathrm{FG} 2$ : $\mathrm{OR}=2.141, p=0.039)$ to develop a severe Cobb angle $\geq 45^{\circ}$ or require a corrective spinal surgery than FG3 patients. The results of the severity threshold outcome were adjusted for the initial Risser score and the initial Cobb angle of main curve.

Looking more specifically at patients from that cohort who have terminated their brace treatment over the observation period ( $n=138$ ) led to a secondary analysis of the associations between brace outcomes (success/failure) and the endophenotype.

The mean Cobb angle was $37.0^{\circ} \pm 15.7^{\circ}$ (median $35.0^{\circ}$ ) at the end of the treatment in the brace subsample, and it 
Table 2 Association between endophenotypes and Cobb angle of main curve at maturity $(n=563)$

\begin{tabular}{llrr}
\hline Independent variables & Reference & Coefficient & $p$ value \\
\hline Model 1 & & & 0.021 \\
FG1 & FG3 & 3.093 & 0.041 \\
FG2 & & 2.412 & \\
Final model & FG3 & & 0.056 \\
FG1 & & 1.425 & 0.050 \\
FG2 & Risser 4-5 (mature) & 1.289 & $<0.001$ \\
Risser 0 & & 5.526 & 0.194 \\
Risser 1 & & 1.435 & 0.285 \\
Risser 2 & & 1.204 & 0.828 \\
Risser 3 & N/A & 0.218 & $<0.001$ \\
Initial Cobb angle of main curve & Thoracic curve & 0.925 & 0.004 \\
Thoracolumbar curve & & -1.941 & 0.005 \\
Lumbar curve & No bracing & -2.083 & $<0.001$ \\
Mainly Boston brace & & 4.047 & $<0.001$ \\
Mainly SpineCor brace & & 5.162 & 0.002 \\
Mainly nighttime brace & N/A & 4.149 & 0.004 \\
Age at skeletal maturity & & 0.639 & \\
\hline
\end{tabular}

Table 3 Association between endophenotypes and severity threshold/ surgery outcome

\begin{tabular}{lrl}
\hline Independent variable & OR & $95 \%$ CI \\
\hline Model 1 & & \\
FG1 & 2.122 & {$[1.175-3.832]$} \\
FG2 & 1.799 & {$[1.034-3.130]$} \\
FG3 & 1.000 & \\
Final model & & \\
FG1 & 2.181 & {$[1.002-4.749]$} \\
FG2 & 2.141 & {$[1.038-4.413]$} \\
FG3 & 1.000 & \\
Risser 0 & 19.561 & {$[7.526-50.845]$} \\
Risser 1 & 5.289 & {$[1.604-17.434]$} \\
Risser 2 & 1.974 & {$[0.543-7.172]$} \\
Risser 3 & 2.228 & {$[0.725-6.848]$} \\
Risser 4-5 (mature) & 1.000 & \\
Maximal initial Cobb angle & 1.227 & {$[1.177-1.280]$} \\
\hline
\end{tabular}

OR: Odds ratio; 95\% CI: 95\% Confidence interval on adjusted OR

was statistically different between the biological endophenotypes. The adjusted model suggests that the endophenotype group was strongly associated with treatment success, even in the presence of the initial Cobb angle and Risser sign in the model (there was no significant effect modification of the studied association by initial Cobb angle, Risser sign, or brace type). The adjusted models were derived for girls only because of potential effect modification in the association by the sex variable, suggested by preliminary log linear analysis, and because of the small sample size for boys precluding stratification in this study. Being classified as FG3
Table 4 Association between brace treatment outcome (success vs failure on the final Cobb angle $\leq 45^{\circ}$ or no surgery criterion) and endophenotypes (FG1, FG2 and FG3) adjusted for initial patient characteristics in girls only $(n=138)$

\begin{tabular}{llll}
\hline Variables & Crude OR & Adjusted OR & $95 \%$ CI \\
\hline \multicolumn{2}{l}{ Biological endophenotype } & & \\
FG1 & 1.0 & 1.0 & \\
FG2 & 1.52 & 1.40 & {$[0.48-4.04]$} \\
FG3 & 7.87 & 9.31 & {$[2.58-33.61]$} \\
Risser sign & & & \\
0 & 1.0 & 1.0 & {$[0.76-0.89]$} \\
1 and 2 & 1.46 & 2.68 & {$[0.76-0.89]$} \\
Cobb angle & 0.83 & 0.82 & \\
\hline
\end{tabular}

OR: Odds ratio; 95\% CI: 95\% Confidence interval on adjusted OR

was 9.31 [2.58-33.61] times more likely to lead to treatment success than failure in comparison to FG1 (Table 4). A similar positive association was observed for the FG2 group, but the value was not statistically significant. Based on the criterion of $<6^{\circ}$ progression in an adjusted model, the FG3 group was 5.63 [2.11-15.05] times more likely to lead to treatment success than failure compared with the FG1 AIS group. There was no significant difference in the treatment outcomes between the FG1 and the FG2 AIS groups.

\section{Discussion}

This study is the first to investigate potential relationships between clinical outcomes in patients with idiopathic scoliosis and endophenotype classification from cell-based essays. 
On a large cohort of 563 patients, our results indicate that patients with FG1 and FG2 endophenotypes may be exposed to a risk of developing a severe curve $\left(>45^{\circ}\right)$ or to undergo surgery as large as twice the risk of those with FG3 endophenotypes. It also appears that the mean curve severity at maturity could be higher in patients with FG1 and FG2 endophenotypes as compared to FG3 patients.

Response to bracing can be affected at the clinical level by many factors including brace design, brace treatment adherence, initial curve severity, and skeletal maturity. In this study, we are proposing the first hypothesis relating brace outcome and intrinsic cellular profile connected to the roles of GPCRs as mechanosensors. This study evaluated patient outcomes based on three biological endophenotypes defined by the differential hypofunctionality of $\mathrm{Gi}$ alpha subunits $[10,11]$ as initially evidenced by a differential dysfunction of melatonin signal transduction [7-9]. Differences between the three endophenotypes acquired through a cell-based assay were identified in the outcomes of brace treatment both for final Cobb and progression criteria. This association suggests a more favorable outcome of bracing for AIS patients classified into the FG3 endophenotype.

Whether the IS patients classified in FG3 endophenotype are genetically predisposed to better respond to brace treatment or are simply less likely to develop progressive forms of IS, remains to be elucidated through a randomized clinical trial. Though the importance of biomechanics and bodily responses to mechanical stimuli is generally well established, the differential processing and interpretation of these signals between affected and healthy individuals remain a largely unexplored frontier. The knowledge of exactly what gene and biochemical pathway responses are altered by mechanotransduction differences in scoliosis could explain the differences observed in bracing outcomes.

Among possible limitations of the present study, although it is worth mentioning that it is a retrospective evaluation of patients from available data in the clinical and radiological files, the study period was long and a great proportion of participants completed the followup. The secondary study is not a controlled study and the sample size is quite limited, leading to wide confidence intervals. However, patients' distribution among the three endophenotypes allowed the investigation of the likelihood of co-occurrence of treatment success with a particular endophenotype. Biological endophenotype classification is invariant over time since it is an intrinsic characteristic of each patient [7-11]. The thresholds between the three endophenotypes are known to be discriminant [7-11]. In particular, misclassification between FG1 and FG3 group is considered very unlikely because there is no overlap in the impedance threshold values between these groups. We have no theoretical or technical reason to believe that a misclassification bias could be differential among the outcome categories. It is therefore unlikely to have affected our conclusion. Misclassification may also occur because of measurement errors in Cobb angles, which was our main outcome measures in the analyses. A sensitivity study was conducted in patients having their final Cobb angle measurement either above or below the $45^{\circ}$ threshold by an interval smaller than the maximum intra-reader difference in Cobb measurements with no significant effect on the studied associations. This potential misclassification error is also believed to be non-differential relative to the biological endophenotypes.

Different types of braces (rigid, non-rigid, and nighttime) were used in this study sample, which reflected the usual practice in our center over the study period. We have also considered brace treatment duration and appropriateness of brace prescription. No significant effect modification by brace type was noted in the regression models. However, quality of brace design and quality of immediate correction are other factors that could have been better evaluated and considered in the analyses as they could have a clear impact on brace treatment success. The proportion of males in the main study sample was coherent with what was expected from the known prevalence IS [2]. For the secondary analysis, the sample size was too limited to investigate the potential effect modification by sex. The measures of association were therefore adjusted for identified control variables in females only.

The determination of clear associations between biological endophenotypes and patient outcomes at maturity/ brace treatment termination may pave the way to establishing opportunities for personalized treatments. These associations may also warrant further investigations in a prospective study including genetic and epigenetic investigations. This approach may open new treatment possibilities by identifying patients that may not respond well to braces and may not benefit from orthopedic treatment. On the other hand, the identification of patients who might respond better to bracing is also important as it may assist the clinicians to support treatment adherence and emphasize the benefits of bracing in certain patient populations.

Acknowledgements We are indebted to the subjects and families who participated in this study. We also thank Ginette Lacroix R.N., for coordinating the study; Ginette Larouche R.N., for her nursing support as well as the nursing teams, all from Sainte-Justine University Hospital Center. Special thanks to Ms. Nikita Cobetto Ing., for her critical reading and review of this manuscript.

Author contributions Study design was performed by MB, FV, FD-B, and AM. All authors contributed to the study conduct. Data collection was performed by M-YA, AF, HL, JJ, J-MM-T, MR-B; Data analysis and interpretation was performed by MB, FV, FD-B, and AM; All authors contributed to the drafting of the manuscript and have read and approved the final manuscript. MB and AM take responsibility for the integrity of the data analysis. 
Funding This work was supported by grants from La Fondation Yves Cotrel de l'Institut de France, Paris, France (to A.M. and H.L.), Paradigm Spine LLC/Fourth Dimension Spine LLC, New York, USA (to A.M.), The Canadian Institutes of Health Research (Grant PP2-99466; to A.M.), and The Depuy Spine Canada Inc. Academic Research Chair in Spinal Deformities, CHU Sainte-Justine Research Center (S.P.), Research Chair in Movement Sciences of the University of Montreal (H.L.).

Data availability The datasets generated and analyzed during the current study are available from the corresponding author on reasonable request.

\section{Compliance with ethical standards}

Conflict of interest This work led to 10 patents (EC1478928, HK1071601, US7989175, US8652791, TW1443337, EC2331962, AU2009301605, ZL200980148704.0, HK1157012, and JP5419986) while other applications are pending on behalf of Sainte-Justine University Hospital (CHU Sainte-Justine). The funding organizations had no role in the design or conduct of the study; in the collection, analysis, or interpretation of data; nor in the preparation, review, or approval of the manuscript. No other potential conflict of interest relevant to this article. Authors have full control of all primary data and agree to allow for review of data if requested.

Ethical approval Informed written consent was obtained from the parents or the legal guardians of all participants, and minors gave their assent. The study was approved by the Institutional Review Board (IRB) of Sainte-Justine University Hospital. All aspects of this research were performed in accordance with the relevant guidelines and regulations.

Informed consent All participants signed informed consent or gave assent regarding publishing their data.

Code availability Not applicable.

Open Access This article is licensed under a Creative Commons Attribution 4.0 International License, which permits use, sharing, adaptation, distribution and reproduction in any medium or format, as long as you give appropriate credit to the original author(s) and the source, provide a link to the Creative Commons licence, and indicate if changes were made. The images or other third party material in this article are included in the article's Creative Commons licence, unless indicated otherwise in a credit line to the material. If material is not included in the article's Creative Commons licence and your intended use is not permitted by statutory regulation or exceeds the permitted use, you will need to obtain permission directly from the copyright holder. To view a copy of this licence, visit http://creativecommons.org/licenses/by/4.0/.

\section{References}

1. Cheng JC, Castelein RM, Chu WC et al (2015) Adolescent idiopathic scoliosis. Nat Rev Dis Primers 1:15030
2. Weinstein S, Dolan L, Wright J, Dobbs M (2013) Effects of bracing in adolescents with idiopathic scoliosis. N Engl J Med 369(16):1512-1521

3. Gorman KF, Julien C, Moreau A (2012) The genetic epidemiology of idiopathic scoliosis. Eur Spine J 21(10):1905-1919

4. Lombardi G, Akoume MY, Colombini A, Moreau A, Banfi G (2011) Biochemistry of adolescent idiopathic scoliosis. Adv Clin Chem 54:165-182

5. Acaroglu E, Bobe R, Enouf J, Marcucio R, Moldovan F, Moreau A (2012) The metabolic basis of adolescent idiopathic scoliosis: 2011 report of the "metabolic" workgroup of the Fondation Yves Cotrel. Eur Spine J 21(6):1033-1042

6. Julien C, Gorman KF, Akoume MY, Moreau A (2013) Towards a comprehensive diagnostic assay for scoliosis. Per Med 10(1):97-103

7. Moreau A, Wang DS, Forget S et al (2004) Melatonin signaling dysfunction in adolescent idiopathic scoliosis. Spine (Phila Pa 1976) 29(16):1772-1781

8. Azeddine B, Letellier K, Wang D, Moldovan F, Moreau A (2007) Molecular determinants of melatonin signaling dysfunction in adolescent idiopathic scoliosis. Clin Orthop Relat Res 462:45-52

9. Akoume MY, Azeddine B, Turgeon I et al (2010) Cell-based screening test for idiopathic scoliosis using cellular dielectric spectroscopy. Spine (Phila Pa 1976) 35(13):E601-E608

10. Akoume M, Franco A, Moreau A (2013) Cell-based assay protocol for the prognostic prediction of idiopathic scoliosis using cellular dielectric spectroscopy. J Vis Exp 80:e50768

11. Akoume MY, Elbakry M, Veillette M et al (2019) A Differential hypofunctionality of Goi proteins occurs in adolescent idiopathic scoliosis and correlates with the risk of disease progression. Sci Rep 9(1): 10074

12. Pope M, Stokes I, Moreland M (1984) The biomechanics of scoliosis. Crit Rev Biomed Eng 11(3):157-188

13. Sarwark JF, Castelein RM, Maqsood A, Aubin CE (2019) The biomechanics of induction in adolescent idiopathic scoliosis: theoretical factors. J Bone Joint Surg Am 101(6):e22

14. Kalichman L, Kendelker L, Bezalel T (2016) Bracing and exercise-based treatment for idiopathic scoliosis. J Bodyw Mov Ther 20(1):56-64

15. Scholz N, Monk KR, Kittel RJ, Langenhan T (2016) Adhesion GPCRs as a putative class of metabotropic mechanosensors. Handb Exp Pharmacol 234:221-247

16. Richards B, Bernstein R, D’Amato C, Thompson G (2005) Standardization of criteria for adolescent idiopathic scoliosis brace studies: SRS committee on bracing and nonoperative management. Spine (Phila Pa 1976) 30(18):2068-2075

17. Nachemson AL, Peterson LE (1995) Effectiveness of treatment with a brace in girls who have adolescent idiopathic scoliosis. A prospective, controlled study based on data from the Brace Study of the Scoliosis Research Society. J Bone Joint Surg Am 77(6):815-822

Publisher's Note Springer Nature remains neutral with regard to jurisdictional claims in published maps and institutional affiliations. 